\title{
Characterization of Polyphenol Oxidase from Walnut
}

\author{
Matthew A. Escobar ${ }^{1}$ and Andrew Shilling \\ Department of Biological Sciences, California State University San Marcos, 333 South Twin Oaks \\ Valley Road, San Marcos, CA 92096 \\ Pine Higgins, Sandra L. Uratsu, and Abhaya M. Dandekar \\ Department of Plant Sciences, University of California Davis, 1 Shields Avenue, Davis, CA 95616
}

\begin{abstract}
AdDitional INDEX words. Juglans regia, phenolic compounds, disease resistance, quinones, L-DOPA, tyrosine
Abstract. The enzyme polyphenol oxidase (PPO) is nearly ubiquitous in Kingdom Plantae and catalyzes the oxidation of phenolic compounds into highly reactive quinones. Although the functional importance of PPO in plants remains uncertain, a putative antipathogen role for walnut (Juglans regia) PPO was posited as early as 1911. However, despite the rich diversity of phenolics present in walnut leaves and hulls, walnut PPO has been little studied since the early 1900s. We cloned a PPO-encoding gene from a walnut pistillate flower cDNA library and designated the gene $j r P P O 1$. Genomic Southern analysis demonstrated that jrPPO1 is the sole PPO gene in walnut. Transgenic tobacco (Nicotiana tabacum) plants expressing jrPPO1 display greater than 10-fold increases in leaf PPO activity compared with wildtype tobacco, demonstrating that $\mathrm{jrPPO1}$ encodes a functional enzyme. The jrPPO1 protein is expressed primarily in the leaves, hulls, and flowers of walnut trees and is not regulated by wounding or methyl jasmonate. To examine whether walnut PPO could affect pathogen resistance, tobacco plants expressing jrPPO1 were challenged with Pseudomonas syringae pv. tabaci. Based on both symptom development and quantitative analyses of bacterial growth in planta, the PPO-expressing plants did not display increased resistance to this pathogen. Leaf extract browning assays indicated that tobacco leaves lack the endogenous phenolic substrates required for significant jrPPO1 activity and quinone production in planta.
\end{abstract}

Polyphenol oxidase (PPO) is a Type 3 copper protein, which catalyzes the oxidation of monophenols or $o$-diphenols to $o$ quinones (Klabunde et al., 1998). PPO-generated quinones are highly reactive and will crosslink with proteins or polymerize, generating dark-colored melanins (Walker and Ferrar, 1998). PPO is of substantial commercial interest as a result of its central role in postharvest browning of a variety of crops, including apple (Malus domestica), potato (Solanum tuberosum), and pineapple (Ananas comosus) (Steffens et al., 1994). In intact plant cells, plastid-localized PPO is physically separated from its phenolic substrates, which reside primarily in the vacuole. Thus, PPO activity is generally observed only on loss of cellular compartmentalization caused by senescence, wounding, or other tissue damage (Steffens et al., 1994).

Characterized PPO isoforms in plants range from $\approx 51$ to 73 $\mathrm{kDa}$ with preproteins often undergoing substantial posttranslational modifications in the form of N-terminal plastid transit peptide processing and $\mathrm{C}$-terminal proteolysis (Thipyapong et al., 2007; VanGelder et al., 1997). All PPOs possess two highly conserved copper-binding domains $(\mathrm{CuA}, \mathrm{CuB})$, each containing several canonical histidine residues that form a $\mathrm{Cu}^{2+}$ binding site (Klabunde et al., 1998). PPO is often isolated from plant tissues in a latent form, which can be activated by a range of treatments, including proteolysis, aging, and incubation with detergents. Unlike animal and fungal PPOs (tyrosinases), many plant PPOs lack monophenol oxidase (cresolase) activity, limiting potential substrates to diphenolic compounds such as

Received for publication 21 Aug. 2008. Accepted for publication 2 Oct. 2008. We thank Tiffany Hauck and Ryan Upham for technical assistance with the PPO browning assays and we are grateful to Brian Staskawicz for providing P. syringae pv. tabaci.

${ }^{1}$ Corresponding author. E-mail: mescobar@csusm.edu. catechol, 3,4-dihydroxyphenylalanine, and chlorogenic acid (Steffens et al., 1994).

PPOs are often, but not exclusively, encoded by multigene families in plants. For example, seven PPO-encoding genes have been identified in tomato (Solanum lycopersicum) and six PPO genes are in the potato genome, whereas PPO appears to be encoded by a single gene in grape (Vitis vinifera) and lettuce (Lactuca sativa) (Thipyapong et al., 2007). Characterized PPOencoding genes in plants are generally $\approx 2 \mathrm{~kb}$ in length and lack introns. They often display distinct tissue-specific and developmental regulation with young leaves, fruit, and flowers possessing the highest PPO transcript levels in several different species (Steffens et al., 1994). In some plants, most notably tobacco, tomato, and hybrid poplar (Populus trichocarpa $\times P$. deltoides), PPO transcription and activity is upregulated in leaves by wounding and the wounding-associated hormones methyl jasmonate and systemin (Constabel and Ryan, 1998; Constabel et al., 2000; Thipyapong and Steffens, 1997).

PPO has several well-defined roles in animals, including skin/exoskeleton pigmentation and cuticle sclerotization (Steffens et al., 1994). In plants, however, the general physiological functions of PPO are substantially less clear, because most PPO research has focused on the agricultural implications of PPO-mediated postharvest browning. Several specialized PPO enzymes have been shown to be involved in the biosynthesis of aurone pigments in snapdragon (Antirrhinum majus) (Nakayama et al., 2001), betalin pigments in pokeweed (Phytolacca americana) (Gandia-Herrero et al., 2005; Joy et al., 1995), and 8-8' linked lignans in creosote bush (Larrea tridentata) (Cho et al., 2003). In addition, a protective function of PPOs has long been hypothesized (Cook et al., 1911; Kosuge, 1969), and several recent studies have provided strong support for a role in plant defense against pathogens and 
insects. In some plant species, insect herbivory strongly induces PPO activity in vivo (Constabel et al., 2000; Ruuhola et al., 2008), and the larvae of forest tent caterpillar (Malacosoma disstria) and common cutworm (Spodoptera litura) display increased mortality and decreased growth when fed leaves of plants overexpressing PPO (Mahanil et al., 2008; Wang and Constabel, 2004). Similarly, viral, bacterial, and fungal challenge can induce PPO activity (Mayer and Harel, 1979), and PPO-overexpressing tomatoes display enhanced resistance to the bacterial pathogen Pseudomonas syringae pv. tomato (Li and Steffens, 2002). Protective effects of PPO have generally been attributed to the generation of reactive quinones, which may 1) possess direct bacteriocidal/insecticidal properties (Vaughn et al., 1988); 2) generate toxic reactive oxygen species through secondary oxidation reactions (Steffens et al., 1994); 3) reduce protein palatability and digestibility by oxidizing nucleophilic amino acids (Felton et al., 1992); and 4) form an impermeable melanin barrier, preventing the spread of pathogen infection (VanGelder et al., 1997).

Walnut possesses an exceptionally abundant and diverse array of polyphenolic compounds (Colaric et al., 2005; Gupta et al., 1972), some of which may play a role in pathogen resistance (Radix et al., 1998; Solar et al., 2006). Thus, walnut presents an especially interesting system for the study of PPO regulation and function. Very early work suggested that "polyatomic phenol oxidase" activity in walnut hulls produced a germicidal fluid that could inhibit bacterial growth (Cook et al., 1911). More recent studies have described the thermostability and basic biochemical properties of PPO activity in walnut hull extracts (Mchedlishvili et al., 2005; Piffaut and Metche, 1991). Overall, however, very little is known about the biochemistry and molecular biology of PPOs in walnut. In this article, we describe the cloning of the single gene encoding PPO in walnut, the regulation of walnut PPO in planta, and the characterization of transgenic plant lines overexpressing walnut PPO.

\section{Materials and Methods}

Plant material and treatments. Walnut (cv. Chandler) tissues were harvested from field-grown trees at the University of California, Davis and were transported to the laboratory on ice. Kernel, pellicle, and hull tissues were removed from $\approx 5$ $\mathrm{cm}$ long mature fruit before hull split. For methyl jasmonate treatments, hulls and leaves were sprayed to runoff with a $0.025 \%$ methyl jasmonate solution in water $/ 0.1 \%$ Triton X-100 (Dow Chemical, Midland, MI) (Constabel and Ryan, 1998). The methyl jasmonate treatment was repeated after $24 \mathrm{~h}$, and tissue was collected $48 \mathrm{~h}$ after the first treatment. Wounding treatments consisted of crushing the leaf midvein with forceps and repeated piercing of the leaf lamina or fruit hull with a 21-G needle. Wounded tissue was collected $48 \mathrm{~h}$ after treatment. Homozygous T2 generation tobacco plants (cv. Petit Havana SR1) transformed with jrPPO1 were grown from seed in a glasshouse under ambient light conditions with air temperature maintained below $28^{\circ} \mathrm{C}$ with an evaporative cooler.

Polyphenol oxidase assays. Total protein was isolated from walnut and tobacco tissues by homogenization in extraction buffer D [50 mM Tris base (pH 8.3), 11\% glycerol, 1\% PEG $8000,0.015 \%$ citric acid monohydrate, $0.010 \%$ cysteine monohydrate, and $0.010 \%$ ascorbic acid]. Homogenization was performed using a 12:1 ratio of buffer $(\mathrm{mL})$ to tissue $(\mathrm{g})$ and 0.5 $\mathrm{g}$ of insoluble PVP per gram tissue was included. After complete homogenization, extracts were centrifuged $(20,000$ $\left.g_{\mathrm{n}}, 4{ }^{\circ} \mathrm{C}, 20 \mathrm{~min}\right)$, and the supernatants were stored at $-80{ }^{\circ} \mathrm{C}$ until use. Protein concentrations were determined by Bradford assay (Bradford, 1976) using bovine serum albumin as a protein standard.

PPO activity assays were performed as described by Constabel and Ryan (1998) using 3,4-dihydroxyphenylalanine (L-DOPA) as a substrate, with dopachrome production monitored spectrophotometrically at $490 \mathrm{~nm}$ (DU 520; Beckman Coulter, Fullerton, CA). The assay buffer contained $100 \mathrm{mM} \mathrm{NaPO}_{4}(\mathrm{pH} 7.0), 0.15 \%$ SDS, $5 \mathrm{mg} \cdot \mathrm{mL}^{-1} \mathrm{~L}-\mathrm{DOPA}$, and $280 \mathrm{U} / \mathrm{mL}$ catalase. Parallel assays were performed in the presence of $2 \mathrm{mM}$ Kojic acid (a specific PPO inhibitor) to verify assay specificity (Constabel and Ryan, 1998).

For in-gel PPO activity staining, total protein (10 to $100 \mu \mathrm{g})$ was separated on a $10 \%$ polyacrylamide gel lacking SDS (a native gel). After electrophoresis, the gel was incubated in a staining solution containing $500 \mathrm{mM} \mathrm{NaPO}_{4}(\mathrm{pH} 7.0), 3.3 \mathrm{mM}$ L-tyrosine (PPO substrate), and $0.16 \mathrm{mM} \mathrm{CuSO}_{4}$ for 1 to $16 \mathrm{~h}$ for color development.

Browning assays were performed essentially as described by Sullivan et al. (2004). Tobacco or walnut leaves were ground to a fine powder in liquid nitrogen using a mortar and pestle, and the tissue powder was extracted into a buffer containing $20 \mathrm{mM}$ Tris base $(\mathrm{pH} 7.5)$ and $100 \mathrm{mM}$ ammonium acetate $(3 \mathrm{~mL}$ of buffer per gram of tissue). Extracts were centrifuged at $20,000 g_{\mathrm{n}}\left(4^{\circ} \mathrm{C}\right)$ for $20 \mathrm{~min}$. Supernatants were then incubated at $22^{\circ} \mathrm{C}$ for 0.5 to $4 \mathrm{~h}$ in the presence or absence of $1 \mathrm{mM}$ L-DOPA, and extract browning was measured spectrophotometrically at $490 \mathrm{~nm}$.

Cloning and Characterization OF JRPPO1. Genomic DNA was isolated from 'Chandler' walnut leaves as previously described (Dellaporta et al., 1983). To isolate a fragment of a PPO-encoding gene from the DNA, degenerate oligonucleotides were designed based on the highly conserved $\mathrm{CuA}$ and $\mathrm{CuB}$ copper-binding domains in plant PPOs. The primers Walt 2 (5'-AAR GAR GCI GAY MGI CCI GCI GGI GCI YTI CCI GTN-3') and Walt 45 (5'-GCR TTY TCR TCR TAR AAR AAR AAI SWI SWR TTI ARC CAR TCI GGR TC-3') were used to polymerase chain reaction (PCR) amplify a $0.8-\mathrm{kb}$ DNA fragment from the genomic DNA. This PPO DNA fragment was cloned into the pCR2.1 vector using a TA Cloning Kit (Invitrogen, Carlsbad, CA). The PPO fragment was subsequently excised from the vector by restriction digestion, gelpurified (Geneclean Kit; MP Biomedicals, Solon, OH), and radioactively labeled using a Boehringer Mannheim Random Primed DNA Labeling Kit (Roche Applied Science, Indianapolis, IN). This probe was used to screen a walnut pistillate flower cDNA library as described subsequently.

To generate a cDNA library, total RNA was extracted from field-collected walnut pistillate flowers using the hot borate method (Wan and Wilkins, 1994). PolyA RNA was isolated from the total RNA using an Oligotex mRNA Maxi Kit (Qiagen, Valencia, CA). Starting with $5 \mu \mathrm{g}$ of polyA RNA, a cDNA library was constructed using a Uni-ZAP/Gigapack II Gold cloning kit (Stratagene, La Jolla, CA) according to the manufacturer's specifications. Plaque hybridization of the cDNA library with the labeled PPO probe (Sambrook et al., 1989) led to the isolation of eight positive phagemids containing cDNA inserts ranging in size from $\approx 1.2$ to $2.0 \mathrm{~kb}$. The largest insert was sequenced and included an 1812-bp open reading frame with high homology to plant polyphenol oxidases (see "Results"). 
To determine the number of PPO genes in the walnut genome, $8 \mu \mathrm{g}$ of walnut genomic DNA was separated on a $0.8 \%$ agarose gel and subjected to Southern hybridization (Sambrook et al., 1989). A digoxigenin-labeled probe consisting of the full-length $\mathrm{jrPPO1}$ open reading frame was generated using a PCR DIG Probe Synthesis Kit (Roche Applied Science) and hybridization was performed in DIG Easy Hyb buffer at $42{ }^{\circ} \mathrm{C}$ (high stringency) or $37^{\circ} \mathrm{C}$ (low stringency) overnight. Washing was performed in $2 \times \mathrm{SSC} / 0.1 \% \mathrm{SDS}$ at room temperature and either $0.5 \times \mathrm{SSC} / 0.1 \% \mathrm{SDS}$ at $68{ }^{\circ} \mathrm{C}$ (high stringency) or $2 \times \mathrm{SSC} / 0.1 \% \mathrm{SDS}$ at $58{ }^{\circ} \mathrm{C}$ (low stringency). Probe hybridization was visualized colorimetrically using a DIG Nucleic Acid Detection Kit (Roche Applied Science).

BINARY VECTOR CONSTRUCTION AND PLANT TRANSFORMATION. Using standard molecular techniques (Sambrook et al., 1989), the jrPPO1 coding sequence was cloned in the sense orientation into the binary vector pDU92.3103 (Tao et al., 1995). The resulting vector, containing jrPPO1 driven by a cauliflower mosaic virus $35 \mathrm{~S}$ promoter, was denoted pDE98.0806. This vector was electroporated into the disarmed Agrobacterium tumefaciens strain C58.C1. Agrobacterium-mediated leaf disc transformation of tobacco (cv. Petit Havana SR1) was performed as previously described (Horsch et al., 1985). Putative transformants were selected on Murashige and Skoog medium containing $100 \mathrm{mg} \cdot \mathrm{L}^{-1}$ kanamycin (Murashige and Skoog, 1962), and transformation was confirmed by histochemical analysis of $\beta$-glucuronidase activity in leaf tissues (data not shown).

Disease Challenge assays. Overnight liquid cultures of $P$. syringae pv. tabaci were grown at $30^{\circ} \mathrm{C}$ in King's B medium supplemented with $100 \mathrm{mg} \cdot \mathrm{L}^{-1}$ rifampicin. Cultures were pelleted by centrifugation at $4000 g_{\mathrm{n}}$ for $10 \mathrm{~min}$ and resuspended in $10 \mathrm{mM} \mathrm{MgCl}_{2}$ to $\mathrm{OD}_{600}=0.5\left[2 \times 10^{8}\right.$ colonyforming units $(\mathrm{cfu}) / \mathrm{mL}]$. The suspension was then diluted $1: 1000$ in $10 \mathrm{mM} \mathrm{MgCl}_{2}$ (to $2 \times 10^{5} \mathrm{cfu} / \mathrm{mL}$ ) and pressureinfiltrated into the abaxial surface of fully expanded tobacco leaves using a needleless syringe (Katagiri et al., 2002). At various time points postinoculation, in planta bacterial populations were determined by sampling the infiltrated leaf tissue using a cork borer and homogenizing the leaf discs in $10 \mathrm{mM}$ $\mathrm{MgCl}_{2}$. Serial dilutions of the homogenates were plated onto King's B medium with $100 \mathrm{mg} \cdot \mathrm{L}^{-1}$ rifampicin and then incubated at $30{ }^{\circ} \mathrm{C}$ for $48 \mathrm{~h}$ for colony counting (Li and Steffens, 2002).

\section{Results}

Previous studies have described PPO activity in walnut hull tissue and heartwood (Cook et al., 1911; Nelson, 1978). To gain a more complete picture of the tissue-level localization of PPO activity in walnut, we isolated protein from nonwoody tissues of field-grown walnut and performed spectrophotometric assays of PPO activity using L-DOPA as a phenolic substrate. As summarized in Figure 1A, PPO activity was detected in all green tissues, including leaves, catin-gel tyrosinase staining. kins, and pistillate flowers. In walnut fruit, high PPO activity was present in hull tissue, but activity was absent within the shell in pellicle and kernel tissues. As a complement to spectrophotometric PPO activity assays, protein extracts were separated using native polyacrylamide gel electrophoresis and subjected to an in-gel PPO activity stain. A single band of melanin deposition with identical electrophoretic mobility was detected in hull, pistillate flower, catkin, and leaf, suggesting that a single common PPO isoform was expressed in these tissues (Fig. 1B). Because the monophenol tyrosine was used as a substrate for the PPO activity stain, our results also demonstrate that walnut PPO possesses cresolase (monophenol oxidase) activity, unlike many plant PPOs (Steffens et al., 1994).

As discussed previously, in some plant species PPO activity is strongly induced $\approx 24$ to $72 \mathrm{~h}$ after wounding or exposure to methyl jasmonate, a regulatory pattern consistent with a function in plant defense (Constabel and Ryan, 1998). To study the regulation of PPO in walnut, we artificially wounded leaf and hull tissue (on tree) or sprayed the tissues with a $0.025 \%$ solution of methyl jasmonate. Forty-eight hours after treatment, total protein was extracted from the tissue and PPO activity assays were performed (Fig. 2). Perhaps unsurprisingly given the high constitutive levels of PPO activity in these tissues, no increase in PPO activity was observed in response to wounding and methyl jasmonate. Considering the reported stability of the PPO enzyme in vivo (Dry and Robinson, 1994; Gooding et al., 2001), this result suggests that walnut polyphenol oxidase is not regulated by wounding and methyl jasmonate, at least over the examined 48-h time interval. Instead, a generally small decrease in PPO activity was observed in both leaf and hull tissues.

To pursue further molecular analyses of PPO in walnut, we attempted to clone the PPO-encoding gene(s) from the walnut genome. Using PCR, we amplified from walnut genomic DNA a $0.8-\mathrm{kb}$ fragment that possessed strong sequence similarity to plant PPOs. This DNA fragment was radioactively labeled and used to probe a cDNA library generated from pistillate flower RNA, allowing the isolation of a phagemid with a $2.0-\mathrm{kb}$ cDNA insert. This insert was fully sequenced, and an 1812-bp open reading frame was identified (Fig. 3). The translated open

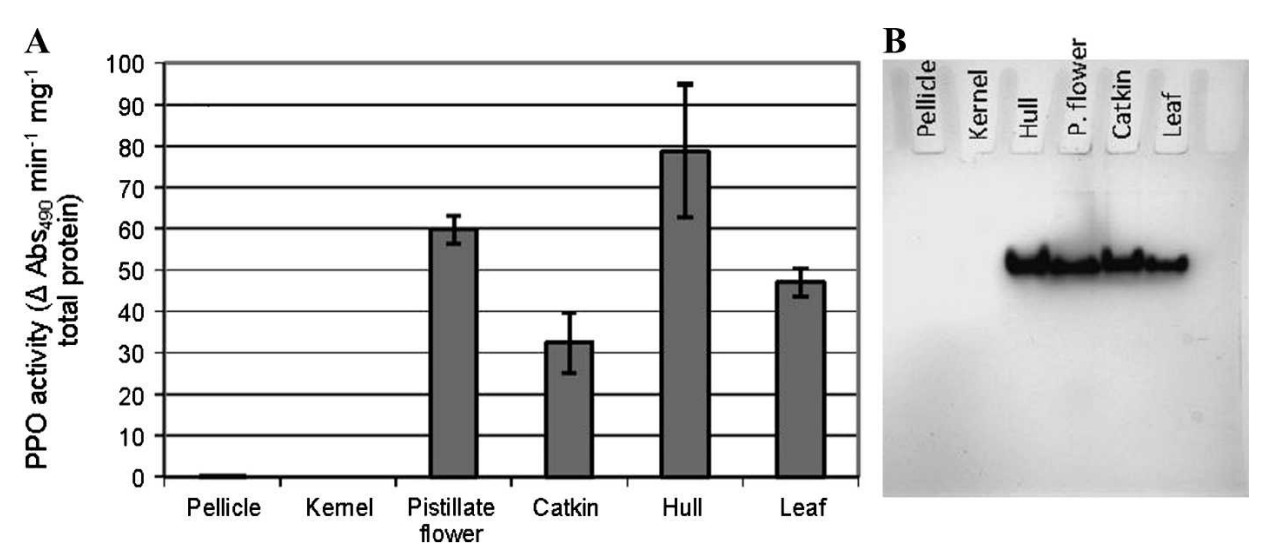

Fig. 1. Tissue localization of polyphenol oxidase (PPO) activity in walnut. (A) PPO activity in walnut tissue extracts was quantified spectrophotometrically, by monitoring the change in absorbance at $490 \mathrm{~nm}\left(\Delta \mathrm{Abs}_{490}\right)$. For each tissue, the mean activity from three biological replicates $( \pm \mathrm{SE})$ is presented. (B) Total protein $(10 \mu \mathrm{g})$ from walnut tissue extracts was separated on a native polyacrylamide gel and PPO activity was determined by 


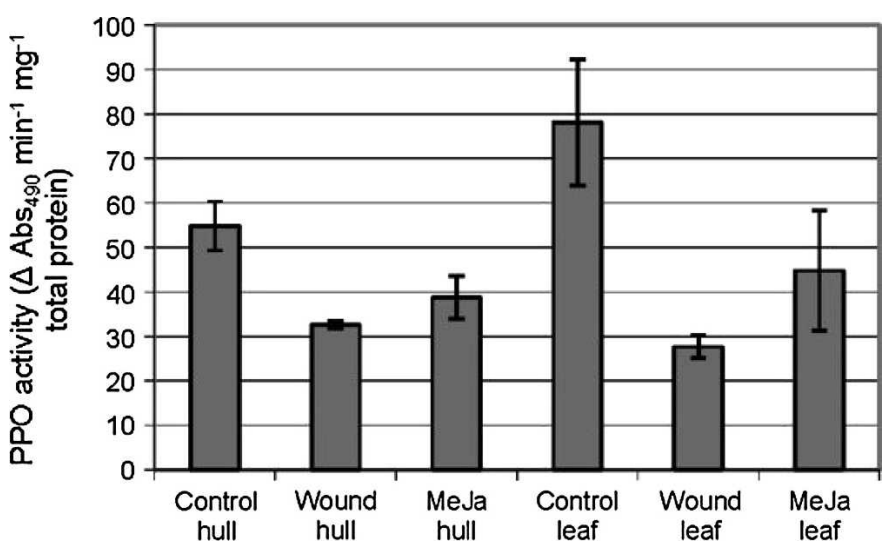

Fig. 2. Regulation of polyphenol oxidase (PPO) in walnut hulls and leaves by wounding and methyl jasmonate (MeJa). Tissues were artificially wounded or sprayed with a $0.025 \%$ solution of methyl jasmonate and harvested $48 \mathrm{~h}$ after treatment. PPO activity in tissue extracts was quantified spectrophotometrically by monitoring the change in absorbance at $490 \mathrm{~nm}\left(\Delta \mathrm{Abs}_{490}\right)$. For each tissue, the mean PPO activity from three biological replicates $( \pm \mathrm{SE})$ is presented.

reading frame possesses greater than $60 \%$ amino acid sequence identity with PPOs from apple, hybrid poplar, grape, and japanese pear (Pyrus pyrifolia) (NCBI accession numbers P43309, AAU12257, CAN61652, and BAB64530, respectively). The N-terminus of the protein shows strong sequence conservation with the N-termini of other plant PPOs and the protein's predicted mass of $67 \mathrm{kDa}$ is typical of plant PPOs, suggesting that the full-length coding sequence has been identified. The gene, denoted jrPPO1, possesses $\mathrm{CuA}$ and $\mathrm{CuB}$ copper-binding domains and is predicted to be chloroplast localized by the subcellular localization prediction programs TargetP and WoLFPSORT (Emanuelsson et al., 1999, 2000; Horton et al., 2007). To determine whether jrPPO1 is a member of a multigene family in walnut, genomic Southern analysis was performed on isolated walnut DNA. Southern blots performed under both high and low stringency hybridization conditions generated single hybridizing bands, indicating that jrPPO1 is the only PPO-encoding gene in the walnut genome (Fig. 4). PCR amplification and sequencing of jrPPO1 from genomic DNA demonstrated that the gene lacks introns (data not shown), which is typical of most plant PPO genes (Thipyapong et al., 2007).

To demonstrate that jrPPOI encodes a functional polyphenol oxidase enzyme, we ectopically expressed the gene in the heterologous host tobacco. jrPPOl was cloned into the binary vector pDU92.3103 (Tao et al., 1995), generating an expression cassette driven by the strong, constitutive cauliflower mosaic virus $35 \mathrm{~S}$ promoter. This binary vector was then used to perform A. tumefaciens-mediated transformation of tobacco. PPO activity was subsequently quantified in leaf protein extracts from three $\mathrm{jrPPO1}$-expressing transgenic tobacco lines. As outlined in Figure 5, several of the transgenic lines displayed five- to 10 -fold increases in spectrophotometrically assayed leaf PPO activity compared with wild-type tobacco plants, demonstrating that jrPPO1 does encode a functional PPO.

If PPOs are important components of plant pathogen defense, then the overexpression of PPO might be expected to increase pathogen resistance. Indeed, Li and Steffens (2002) demonstrated that overexpression of a potato PPO in tomato atggettctetcteggettcetcctcccacctagaagcaccaacatggtggctgccace $\begin{array}{llllllllllllllllllll}M & A & S & L & L & A & S & S & S & H & P & R & S & T & N & M & V & A & A & T\end{array}$ acgaccattcgcaccectcttecetcectgtttectagaaatacacaagtttctaaa

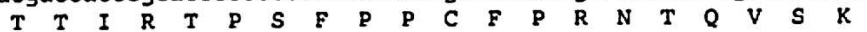
gttggaaagcaataccaccaccttactactagagtggcatgcagagctacaaatggtgac $\begin{array}{llllllllllllllllllll}V & G & K & Q & Y & H & H & L & T & T & R & V & A & C & R \nabla & A & T & N & G & D\end{array}$ caacaaaatattaataatggacaaaatcctcaagggaagttcgatagaagagatgtacte $\begin{array}{llllllllllllllllllll}Q & Q & N & I & N & N & G & Q & N & P & Q & G & K & F & D & R & R & D & V & L\end{array}$ cttggcctcggaggcatgtatggtgctgccaatctctgcaatgacccgtttgcgetggca

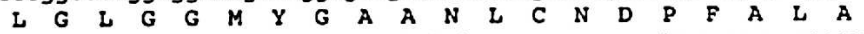
gatccggtatccgcgecggaattaacactgtgtagcgaggcagacetcceagcaggcgca $\begin{array}{llllllllllllllllllll}D & P & V & S & A & P & E & L & T & L & C & S & E & A & D & L & P & A & G & A\end{array}$ cttccagtcaattgergcccacctacatccaagaagatcaaagactttgttttacctage

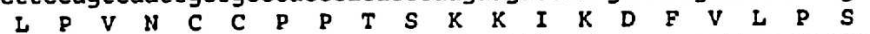
caaacactccettacgtgttaggectgcggetcattggttgacaacgattacatagcc

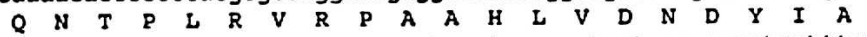
aaatataacaaaggcattgagctcatgaagtccetcceggetgatgaccegcgtagttte $\begin{array}{llllllllllllllllllll}K & Y & N & K & G & I & E & L & M & K & S & L & P & A & D & D & P & R & S & E\end{array}$ acccaacaagetaatgtccactgcgcctactgcgacggggettacacacaagtcggtt tt $\begin{array}{llllllllllllllllllll}T & Q & Q & A & N & V & H * & C & A & Y & C & D & G & A & Y & T & Q & V & G & F\end{array}$ cagacttgagcctccaagttcacgaatgtrggctcttcttccattccatcgctactac

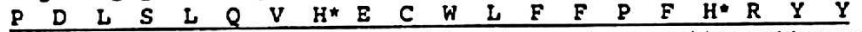
gtgracttctegagaaatattgggcaagttgattggcgatcccaccttegcetgca \begin{tabular}{llllllllllllllllllll}
$V$ & $Y$ & $F$ & $F$ & $E$ & $K$ & $I$ & $L$ & $G$ & $K$ & $L$ & $I$ & $G$ & $D$ & $P$ & $T$ & $F$ & $A$ & $L$ & $P$ \\
\hline
\end{tabular} ttctggaactgggactccctectggtatgcaattgccatccttgtatgctgtctccaac

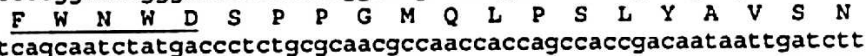
tcagcaatctatgaccetctgcgcaacgccaaccaccagccaccgacaataattgatctt gactacggcgagaccagcgagtcaacgacaacaacagatcaagtacctagcaacctcaaa D Y G E T S E S T T T T D Q V P S N L K atcatgtaccggcagatggrgtccggegccaagaacctacgctatttttcggcagcect

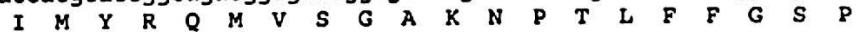
tatcgggctggggatgaacctgacccaggtgctggcacaatcgagagcactcccacaat $Y \quad R$ A G D E $P$ D $P$ G A $G$ T $I$ E $S$ T aatatccacctatggaccggtgacgacacccaacctaatatcgagaacatggggaacttc

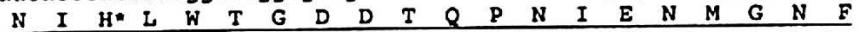

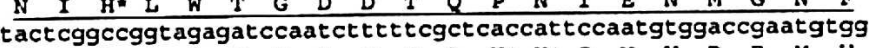

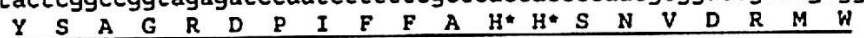
accatatggaaaacattaggagggaaacgaaaagataccacagacccagattggttgaac $\begin{array}{llllllllllllllllllll}T & I & W & K & T & L & G & G & K & R & K & D & I & T & D & P & D & W & L & N\end{array}$ tcctcatttetctetatgatgaaaatgcagatcctgttcgtgttaaggttaaggactge $\begin{array}{llllllllllllllllllll}S & S & F & F & F & Y & D & E & N & A & D & P & V & R & V & K & V & K & D & C\end{array}$ gttgataacactaagetgagatatgtetatcaagatgtggagattccatggetaaagace $\begin{array}{llllllllllllllllllll}V & D & N & T & K & I & R & Y & V & Y & Q & D & V & E & I & P & W & L & K & T\end{array}$ aagccgacacctcgtaaatctagggttaagaaagtagcgaaagcctt tcagccggacat

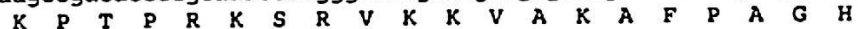
ggtggtgtagcacaagcggetgaaacatcgagcgttaagttrccgategttttggacaag G G V A Q A A E T S S V K gtgataagtactgttgtcgetaggeccaagaaatcgaggagcaagaaagaaaaggacgac $\begin{array}{lllllllllllllllllll}V & I & S & T & V & A & R & P & K & K & S & R & S & K & K & E & K & D & D\end{array}$ gaggaagaagttttagtgattgagggtattgaggttgagagagatattccagtgaagttt

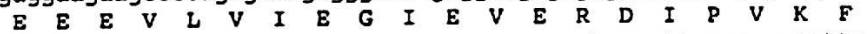
gatgtttttatcaacgacgaggatgacgcaccaaccgggcctggaattaacacggagttc $\begin{array}{llllllllllllllllllll}D & V & F & I & N & D & E & D & D & A & P & T & G & P & G & I & N & T & E & F\end{array}$ gcaggaagctttgtcagcgtgcegcagcagaagcagacgaagaagaagaaaacttacctg

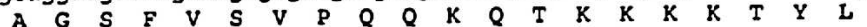
aggataggaatctctgacttgttggaagacttgggagctgaagatgatgactccgtggtg $R$ I G I S D L I E D L G A $E$ D $D$ D $S$ V V gtgactttggtacccoggttegggaaagggaaggccatcattggtgggatcaagattgtg $\begin{array}{llllllllllllllllllll}V & I & L & V & P & R & F & G & K & G & K & A & I & I & G & G & I & K & I & V\end{array}$ cttatcggttga

L I G -

Fig. 3. Nucleotide and predicted amino acid sequence of the walnut polyphenol oxidase (PPO) gene $\mathrm{jrPPO1}$. The $\mathrm{jrPPO1}$ open reading frame is $1812 \mathrm{nt}$ and encodes a $67 \mathrm{kDa}$ preprotein possessing greater than $60 \%$ amino acid sequence identity to a variety of previously identified plant PPOs. The $\mathrm{CuA}$ and $\mathrm{CuB}$ motifs are underlined, with copper-binding histidine residues denoted with an asterisk. The putative chloroplast transit peptide cleavage site, as predicted by ChloroP (Emanuelsson et al., 1999), is denoted with an arrowhead.

substantially increased resistance to the bacterial pathogen $P$. syringae pv. tomato. Thus, we examined whether expression of $\mathrm{jrPPO1}$ in tobacco increased resistance to P. syringae pv. tabaci, a causal agent of angular leaf spot. Leaves from two transgenic tobacco lines displaying the highest PPO activity (10D, 12D) were pressure-infiltrated with a $2 \times 10^{5} \mathrm{cfu} / \mathrm{mL}$ suspension of $P$. syringae pv. tabaci, and bacterial growth was monitored by plating diluted leaf extracts at 0 to $4 \mathrm{~d}$ 


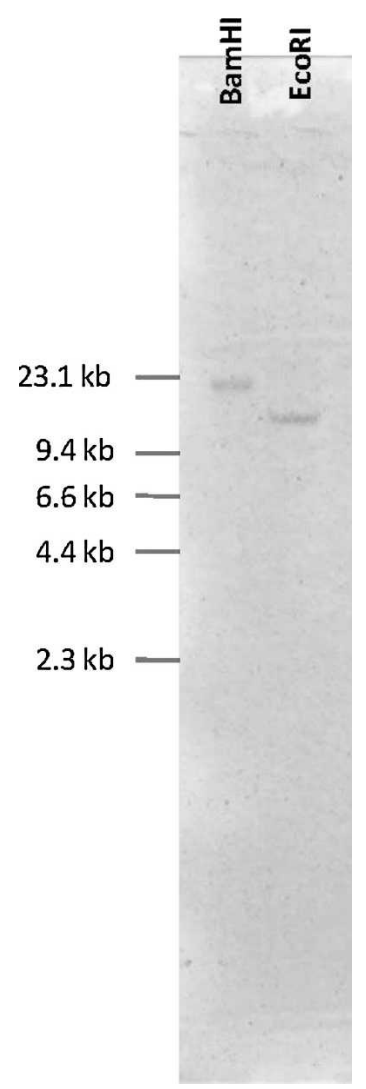

Fig. 4. Southern analysis of walnut genomic DNA to determine polyphenol oxidase (PPO) gene copy number. Eight micrograms of walnut genomic DNA was digested with the BamHI and EcoRI restriction enzymes and size separated on an agarose gel. After capillary transfer to a positively charged nylon membrane, hybridization was performed with a full-length $\mathrm{jrPPO1}$ probe labeled with digoxigenin. Because neither BamHI nor EcoRI recognition sites are present in the jrPPOI sequence, single bands in the Southern analysis indicate a single copy gene. Similar results were obtained using both high and low stringency hybridization conditions (see "Materials and Methods").

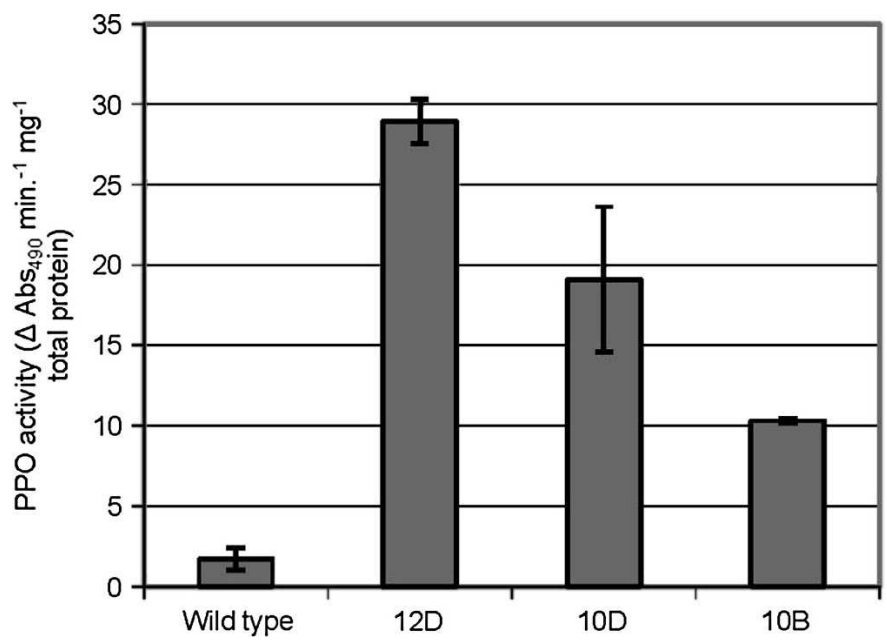

Fig. 5. Polyphenol oxidase (PPO) activity in leaf extracts from transgenic tobacco plants $(12 \mathrm{D}, 10 \mathrm{D}, 10 \mathrm{~B})$ expressing the walnut PPO gene jrPPO1. PPO activity was quantified spectrophotometrically by monitoring the change in absorbance at $490 \mathrm{~nm}\left(\Delta \mathrm{Abs}_{490}\right)$. For each line, the mean activity from three biological replicates $( \pm \mathrm{SE})$ is presented. postinoculation. However, unlike the findings of Li and Steffens (2002), both disease symptoms and bacterial growth in planta were unchanged in the jrPPO1-expressing plants (Fig. 6).

Browning assays performed on tobacco leaf extracts showed that extract browning (caused by PPO-mediated quinone formation and polymerization into melanin) occurred only in the presence of exogenously added phenolic substrate (LDOPA) (Fig. 7). In contrast, walnut leaf extracts display rapid browning in the absence of exogenously added substrates, demonstrating that endogenous phenolic substrates are abundant in walnut leaves (Fig. 7). Thus, any potential antipathogen effects of $\mathrm{jrPPO1}$ may be limited by the lack of appropriate phenolic substrates in the tobacco experimental system.

\section{Discussion}

Although the use of walnut extracts as hair dyes and skin colorants has a long history (Bittner, 2006), little has been learned about the specific enzymes that underlie these browning processes since Cook et al.'s (1911) initial description of PPO activity in walnut hulls. Our data demonstrate that Cook's "poly-atomic phenol oxidase" is encoded by a single gene, jrPPO1, which is constitutively expressed in all green, herbaceous tissues of walnut. Strikingly, PPO-specific activity in walnut tissue extracts is extremely high compared with characterized PPO activity in other plant species. Basal PPO
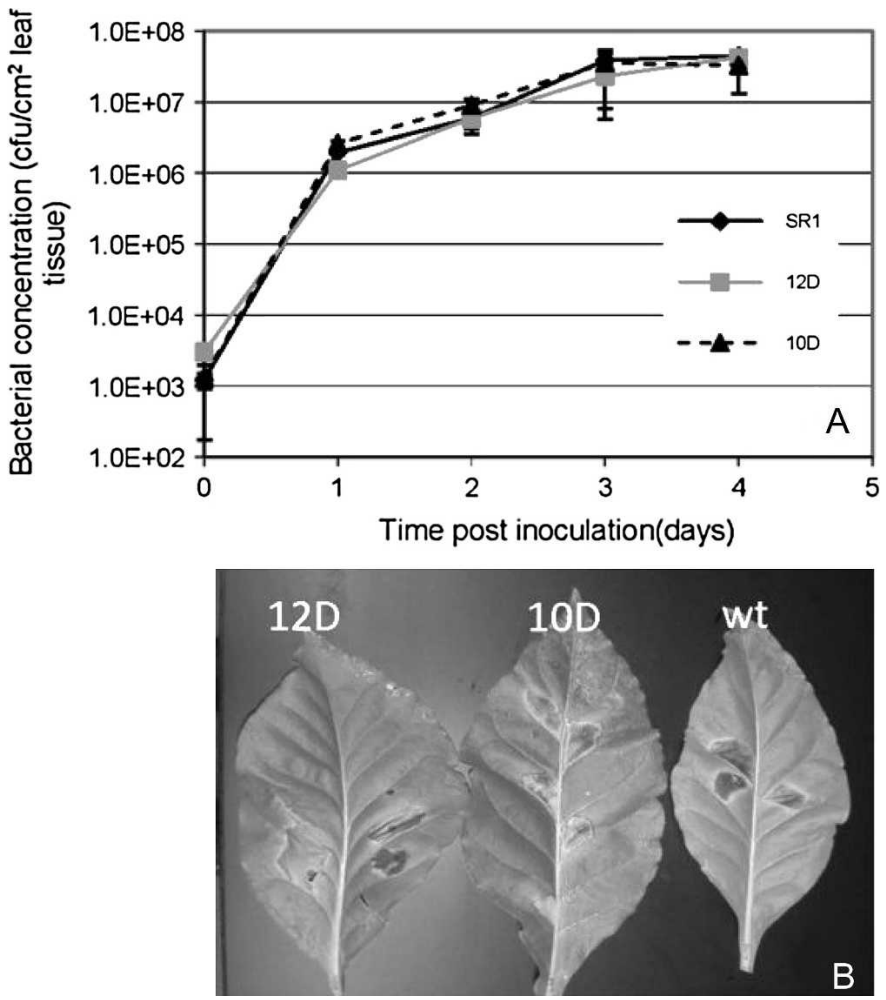

Fig. 6. Expression of the walnut polyphenol oxidase (PPO) gene $\mathrm{jrPPO1}$ in transgenic tobacco plants does not confer increased resistance to the bacterial pathogen Pseudomonas syringae pv. tabaci. Fully expanded leaves of wildtype (wt) and $j r P P O 1$-expressing transgenic tobacco lines (10D, 12D) were infiltrated with a $2 \times 10^{5} \mathrm{cfu} / \mathrm{mL}$ suspension of $P$. syringae pv. tabaci. (A) In planta bacterial populations were quantified by plating diluted extracts from inoculated tissue. Data points represent mean bacterial concentrations $\pm \mathrm{SE}$ for three biological replicates. (B) Disease symptom development in the leaves of wild-type, 10D, and 12D tobacco plants is shown. 


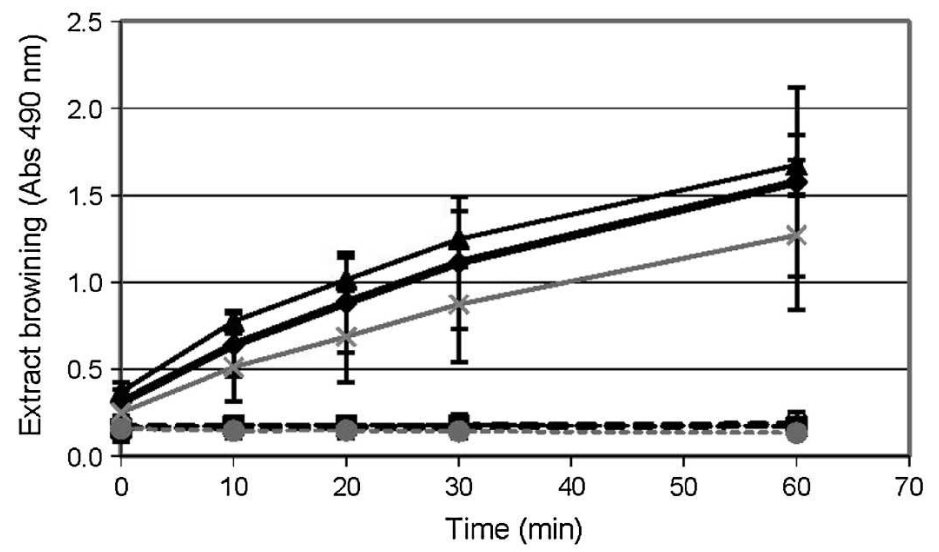

A

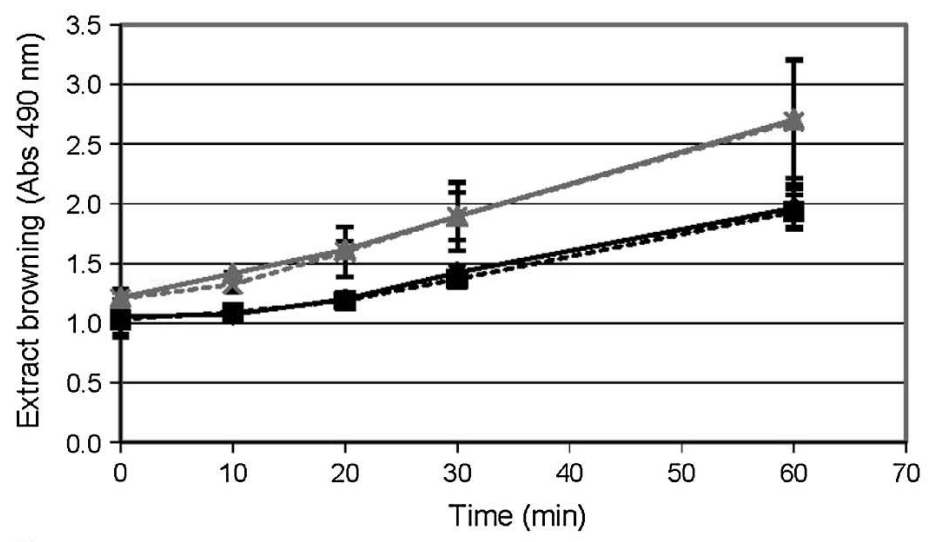

B

Fig. 7. Tobacco leaves lack endogenous substrates for the walnut polyphenol oxidase $j r P P O 1$. Freshly isolated protein extracts from $1 \mathrm{~g}$ of tobacco leaves (A) or walnut hulls/leaves (B) were incubated in the presence or absence of 3,4-dihydroxyphenylalanine (L-DOPA) for the time periods indicated. Extract browning was monitored by measuring absorbance at $490 \mathrm{~nm}$. Data points represent mean absorbance $\pm \mathrm{sD}$ from two biological replicates. Insets demonstrate typical results after a 60-min incubation with + and -indicating the presence and absence of L-DOPA, respectively.

activity in walnut leaves was more than 10 times higher than the PPO activity reported in the leaves of wounded (induced) tobacco and hybrid poplar plants and is comparable to the highest PPO activity observed in a survey of 18 crop plants (leaves of tomato plants treated with methyl jasmonate) (Constabel and Ryan, 1998). To our knowledge, high constitutive expression of PPO has been described only in soybean (Glycine max) and willow (Salix spp.) and is in contrast to the moderate or dramatic wound induction of PPO in most plant species (Constabel and Ryan, 1998). Although significant evidence supports a bacteriocidal and/or insecticidal role for PPO in species such as hybrid poplar and tomato, constitutive expression is not generally associated with disease and pest resistance genes as a result of large associated fitness costs (Heidel et al., 2004). Thus, the native physiological role of highly expressed PPOs in species such as walnut, willow, and soybean remains unclear.

As described, the expression of jrPPO1 in transgenic tobacco plants increased leaf PPO activity greater than 10 -fold (line 12D) but had no effect on susceptibility to the pathogen P. syringae pv. tabaci (Figs. 5 and 6). However, it is not PPO protein per se, but the reactive quinones generated by

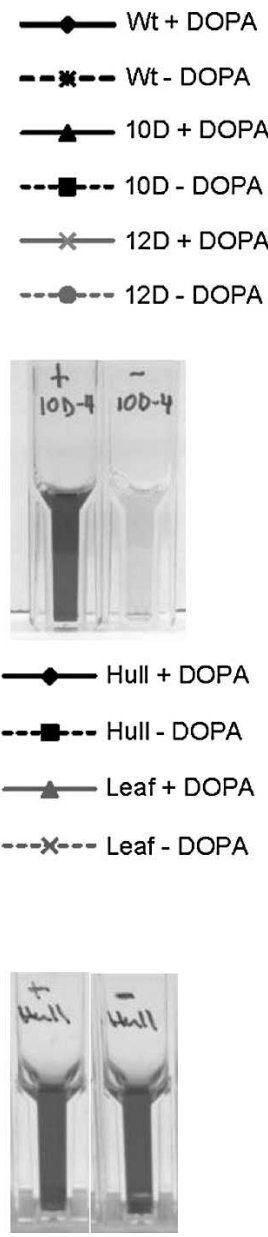

PPO activity, that are thought to play a role in inhibiting pathogen/insect growth in some plants (Steffens et al., 1994). Thus, both PPO and abundant phenolic substrates are presumably required for PPO to fulfill a role in defense, and these endogenous phenolic substrates are clearly lacking in tobacco leaves under the tested conditions. This lack of appropriate phenolic substrates in tobacco leaves likely explains the contrast between our results and the work of $\mathrm{Li}$ and Steffens (2002), who found that overexpression of PPO in tomato increased bacterial disease resistance. A recent examination of quinone-mediated inhibition of proteolysis during ensiling of alfalfa parallels the results of our studies with jrPPO1-expressing tobacco. In this work, expression of a red clover (Trifolium pratense) PPO in alfalfa (Medicago sativa) did not inhibit postharvest proteolysis unless phenolic substrates were exogenously supplied, reflecting a lack of native PPO substrates in alfalfa plants (Sullivan et al., 2004). Thus, transgenic approaches to the use of PPO in plant improvement are probably best applied between closely related species [as in Li and Steffens' (2002) work with potato and tomato], in which appropriate phenolic substrates are more likely to be present in the plant host.

To continue our examination of the potential physiological functions of PPO in walnut (where jrPPO1 substrates are abundant, see Fig. 7), we recently generated transgenic walnut plants expressing a selfcomplementary jrPPO1 transgene designed to silence PPO in planta. Future studies characterizing the basic physiology, growth, and susceptibility to disease and insect pests of these PPO-silenced walnuts should provide valuable further information about the functional role of the highly abundant PPO enzyme in walnut.

\section{Literature Cited}

Bittner, S. 2006. When quinones meet amino acids: Chemical, physical and biological consequences. Amino Acids 30:205224.

Bradford, M.M. 1976. Rapid and sensitive method for quantitation of microgram quantities of protein utilizing the principle of protein-dye binding. Anal. Biochem. 72:248-254.

Cho, M.H., S.G.A. Moinuddin, G.L. Helms, S. Hishiyama, D. Eichinger, L.B. Davin, and N.G. Lewis. 2003. (+)-Larreatricin hydroxylase, an enantio-specific polyphenol oxidase from the creosote bush (Larrea tridentata). Proc. Natl. Acad. Sci. USA 100:10641-10646. 
Colaric, M., R. Veberic, A. Solar, M. Hudina, and F. Stampar. 2005. Phenolic acids, syringaldehyde, and juglone in fruits of different cultivars of Juglans regia L. J. Agr. Food Chem. 53:6390-6396.

Constabel, C.P. and C.A. Ryan. 1998. A survey of wound- and methyl jasmonate-induced leaf polyphenol oxidase in crop plants. Phytochemistry 47:507-511.

Constabel, C.P., L. Yip, J.J. Patton, and M.E. Christopher. 2000. Polyphenol oxidase from hybrid poplar. Cloning and expression in response to wounding and herbivory. Plant Physiol. 124:285-295.

Cook, M.T., H.P. Bassett, F. Thompson, and J.J. Laubenhaus. 1911. Protective enzymes. Science 33:624-629.

Dellaporta, S., J. Wood, and J.B. Hicks. 1983. A plant DNA minipreparation: Version II. Plant Mol. Biol. Rpt. 1:19-21.

Dry, I.B. and S.P. Robinson. 1994. Molecular-cloning and characterization of grape berry polyphenol oxidase. Plant Mol. Biol. 26:495-502.

Emanuelsson, O., H. Nielsen, S. Brunak, and G. von Heijne. 2000. Predicting subcellular localization of proteins based on their Nterminal amino acid sequence. J. Mol. Biol. 300:1005-1016.

Emanuelsson, O., H. Nielsen, and G. Von Heijne. 1999. ChloroP, a neural network-based method for predicting chloroplast transit peptides and their cleavage sites. Protein Sci. 8:978-984.

Felton, G.W., K.K. Donato, R.M. Broadway, and S.S. Duffey. 1992. Impact of oxidized plant phenolics on the nutritional quality of dietary protein to a noctuid herbivore, Spodoptera exigua. J. Insect Physiol. 38:277-285.

Gandia-Herrero, F., J. Escribano, and F. Garcia-Carmona. 2005. Betaxanthins as substrates for tyrosinase: An approach to the role of tyrosinase in the biosynthetic pathway of betalains. Plant Physiol. 138:421-432.

Gooding, P.S., C. Bird, and S.P. Robinson. 2001. Molecular cloning and characterisation of banana fruit polyphenol oxidase. Planta 213:748-757.

Gupta, S.R., T.R. Seshadri, and B. Ravindra. 1972. Polyphenols of Juglans nigra. Phytochemistry 11:2634-2636.

Heidel, A.J., J.D. Clarke, J. Antonovics, and X.N. Dong. 2004. Fitness costs of mutations affecting the systemic acquired resistance pathway in Arabidopsis thaliana. Genetics 168:2197-2206.

Horsch, R.B., J.E. Fry, N.L. Hoffmann, D. Eichholtz, S.G. Rogers, and R.T. Fraley. 1985. A simple and general method for transferring genes into plants. Science 227:1229-1231.

Horton, P., K.J. Park, T. Obayashi, N. Fujita, H. Harada, C.J. AdamsCollier, and K. Nakai. 2007. WoLF PSORT: Protein localization predictor. Nucleic Acids Res. 35:W585-W587.

Joy, R.W., M. Sugiyama, H. Fukuda, and A. Komamine. 1995. Cloning and characterization of polyphenol oxidase cDNAs of Phytolacca americana. Plant Physiol. 107:1083-1089.

Katagiri, F., R. Thilmony, and S.Y. He. 2002. The Arabidopsis thaliana-Pseudomonas syringae interaction, p. 1-35. In: Somerville, C. and E. Meyerowitz (eds.). The arabidopsis book. Amer. Soc. Plant Biologists, Rockville, MD.

Klabunde, T., C. Eicken, J.C. Sacchettini, and B. Krebs. 1998. Crystal structure of a plant catechol oxidase containing a dicopper center. Nat. Struct. Biol. 5:1084-1090.

Kosuge, T. 1969. Role of phenolics in host response to infection. Annu. Rev. Phytopathol. 7:195-222.

Li, L. and J.C. Steffens. 2002. Overexpression of polyphenol oxidase in transgenic tomato plants results in enhanced bacterial disease resistance. Planta 215:239-247.

Mahanil, S., J. Attajarusit, M.J. Stout, and P. Thipyapong. 2008. Overexpression of tomato polyphenol oxidase increases resistance to common cutworm. Plant Sci. 174:456-466.

Mayer, A.M. and E. Harel. 1979. Polyphenol oxidases in plants. Phytochemistry 18:193-215.

Mchedlishvili, N.I., N.T. Omiadze, L.K. Gulua, T.A. Sadunishvili, R.K. Zamtaradze, M.O. Abutidze, E.G. Bendeliani, and G.I. Kvesitadze.
2005. Thermostabilities of plant phenol oxidase and peroxidase determining the technology of their use in the food industry. Appl. Biochem. Microbiol. 41:145-149.

Murashige, T. and F. Skoog. 1962. A revised medium for rapid growth and bio assays with tobacco tissue cultures. Physiol. Plant. 15:473-479.

Nakayama, T., T. Sato, Y. Fukui, K. Yonekura-Sakakibara, H. Hayashi, Y. Tanaka, T. Kusumi, and T. Nishino. 2001. Specificity analysis and mechanism of aurone synthesis catalyzed by aureusidin synthase, a polyphenol oxidase homolog responsible for flower coloration. FEBS Lett. 499:107-111.

Nelson, N.D. 1978. Xylem ethylene, phenol-oxidizing enzymes, and nitrogen and heartwood formation in walnut and cherry. Can. J. Bot. 56:626-634.

Piffaut, B. and M. Metche. 1991. Properties of peroxidase and polyphenol oxidase in natural complexes from walnuts (Juglans regia) and in active DL-DOPA copolymers. J. Sci. Food Agr. 57:493-506.

Radix, P., C. Bastien, C. Jay-Allemand, G. Charlot, and F. SeigleMurandi. 1998. The influence of soil nature on polyphenols in walnut tissues: A possible explanation of differences in the expression of walnut blight. Agronomie 18:627-637.

Ruuhola, T., S.Y. Yang, V. Ossipov, and E. Haukioja. 2008. Foliar oxidases as mediators of the rapidly induced resistance of mountain birch against Epirrita autumnata. Oecologia 154:725-730.

Sambrook, J., E.F. Fritsch, and T. Maniatis. 1989. Molecular cloning: A laboratory manual. Cold Spring Harbor Laboratory Press, Cold Spring Harbor, NY.

Solar, A., M. Colaric, V. Usenik, and F. Stampar. 2006. Seasonal variations of selected flavonoids, phenolic acids and quinones in annual shoots of common walnut (Juglans regia L.). Plant Sci. 170:453-461.

Steffens, J.C., E. Harel, and M.D. Hunt. 1994. Polyphenol oxidase, p. 275-312. In: Ellis, B.E. (ed.). Genetic engineering of plant secondary metabolism. Plenum Press, New York, NY.

Sullivan, M.L., R.D. Hatfield, S.L. Thoma, and D.A. Samac. 2004. Cloning and characterization of red clover polyphenol oxidase cDNAs and expression of active protein in Escherichia coli and transgenic alfalfa. Plant Physiol. 136:3234-3244.

Tao, R., S.L. Uratsu, and A.M. Dandekar. 1995. Sorbitol synthesis in transgenic tobacco with apple cDNA-encoding NADP-dependent sorbitol-6-phosphate dehydrogenase. Plant Cell Physiol. 36: $525-532$.

Thipyapong, P. and J.C. Steffens. 1997. Tomato polyphenol oxidaseDifferential response of the polyphenol oxidase $F$ promoter to injuries and wound signals. Plant Physiol. 115:409-418.

Thipyapong, P., M.J. Stout, and J. Attajarusit. 2007. Functional analysis of polyphenol oxidases by antisense/sense technology. Molecules 12:1569-1595.

VanGelder, C.W.G., W.H. Flurkey, and H.J. Wichers. 1997. Sequence and structural features of plant and fungal tyrosinases. Phytochemistry 45:1309-1323.

Vaughn, K.C., A.R. Lax, and S.O. Duke. 1988. Polyphenol oxidase-The chloroplast oxidase with no established function. Physiol. Plant. 72:659-665.

Walker, J.R.L. and P.H. Ferrar. 1998. Diphenol oxidases, enzymecatalysed browning and plant disease resistance. Biotechnol. Genet. Eng. Rev. 15:457-498.

Wan, C.Y. and T.A. Wilkins. 1994. A modified hot borate method significantly enhances the yield of high quality RNA from cotton (Gossypium hirsutum L.). Anal. Biochem. 223:7-12.

Wang, J.H. and C.P. Constabel. 2004. Polyphenol oxidase overexpression in transgenic Populus enhances resistance to herbivory by forest tent caterpillar (Malacosoma disstria). Planta 220: $87-96$. 